\title{
Author Index Vol. 22, 1995
}

\section{Abbott, R. 309 Adelson, P.D. 174 Amin-Hanjani, S. 299 Andermann, F. 167 Ang, L.-C. 321 Apfelbaum, R. 147 Axtell, R.A. 65}

Barone, CM. 162 Baumgartner, J.E. 274 Becker, L.E. 104 Bharucha, B.A. 328 Bierbrauer, K.S. 137 Biglan, A.W. 270 Black, P.McL. 174,197 Boyd, M. 141 Boyett, J. 1 Brockmeyer, D. 147 Brownlee, R.D. 315 Brunberg, J.A. 65 Bruner, J.M. 274 Burger, P.C. 189 Burke, M.J. 241

Campbell, J.W. 270 Carey,|L. 147 Casey,|R. 321 Cochrane D.D. 136 Cohen,|M.E. 189 Comair, Y.G. 181 Constantini, S. 111,303 Costarino, D. 81 Cuka, G.M. 101

Daneshvar, H. 255 Das, S.K. 57 Dauser, R.C. 65 Deshmukh, C.T. 328 Dirks, P. 204 Dold, O.N.R. 315 Douglas Cochrane, D. 136,141 Douglass, E.C. 289 Duffner, P.K. 189 Duhaime, A.C. 81

Ellis, T. 266

Epstein, FJ. 111,223,309 Estes, M.L. 181 Evans, D. 255

Farmer, J.-P. 167 Flodmark, CO. 141 Fontanesi, J. 289 Friedman, H.S. 189 Fukuyama, K. 204

Gabriel,|K. 96 George, D.H. 321 Geyer, J.R. 1 Goodrich, J.T. 247 Gordon McComb, J. 166 Goumnerova, L.C 197 Gupte, G.L. 328

Hada, H. 108 Hahn, J.F. 181 Haines, D.E. 57 Harkness, W.J. 235 Hayward, R.D. 235 Heideman, R.L. 289 Hellbusch, L.C 101 Helmers, S.L. 174 Herzog, CE. 274 Hinton, D.R. 104 Hoffman, H.J. 213 Holmes, G.L. 174 Horowitz, M.E. 189 Hubbard, S.L. 204 Humphreys, R.P. 38, 136, 223, 240,265,314,320

Inagawa, T. 108 Irani, S.F. 328 Israel,,Z.H. 210

James, H.E. 189,248 Jimenez, D.F. 162 Johnson, D.L. 115 Jones, B.M. 235

Kanev, P.M. 137 Katoh, Y. 108 Katz, R. 248 Kaufman, B.A. 74 Kawecki, S. 281 Kestle, J.R.W. 255 Khajavi, K. 181 Kiburz, J. 96 Kovnar, E. 289 Kramer,U. 174 Krischer, J.P. 189 Kun, L, E. 189,289 Kuo, M.F. 158 Kuttesch, J.F. 289

Leahu, D. 309

Lee, M. 251,309

Leeds, N.E. 274

Leland Albright, A. 1,29,270

Levy, L.M. 115

Liu, H.-M. 158

Loeffler, J.S. 197 López-Corella, E. 49 Losken, H.W. 270 Lowry, P.A. 8

McComb, J.G. 15,166 McGraü, K. 228 McLone, D.G. 131 McMillan, T. 132 Madsen, J.R. 174 Malcolm, G.P. 235 Manhoff, D.T. 214 Matsuzawa, K. 204 Meagher-Villemure, K. 167 Meltzer, H. 248 Menezes, A.H. 88 Mickle, J.P. 266 Mikati, M. 174 Miller,D.C 303 Milstein, J.M. 1 Mitchell, P. 20 Montes, J.L. 167 Moran, CJ. 74 Mori, K. 120 Muhlbauer, M. 289 Mulhern, R.K. 189,289 Muller, M. 204 Muraszko, K.M. 65 Myles, S.T. 315

Nishimura, T. 120

Ochs, JJ. 289 Ogilvy, CS. 228 O'Gorman, A.M. 167 O’Neill, O.R. 20

Palmer,|J. 181 Parent,|A.D. 57,132 Park, T.S. 74,265 Patrice,|S.J. 197 Pediatric Oncology Group 1 í Piatt, J.H., Jr. 20 Pittman, T. 96 Pollack, I.F. 29,281 Pomeranz, S. 210 Prayson, RA. 181

Quisling, R. 266

Raffel, C 15 Rees, H. 321 Reigel, D.H. 223 Rekate, H.L 189 Resnick, D.K. 29

Rezai, A.R. 251 Riviello, J.J. 174 Robertson, P.L. 65 Rockoff, M.A. 174 Rojiani, A.M. 266 Rollins, N.K 8 Roman-Goldstein, S. 20 Rorke, L.B. 1,214 Rosenblatt, B. 167 Rueda-Franco, F. 49 , 56 Rutka, J.T. 204 Ryken, T.C 88

Sanford, R.A. 189,289 Sathi, S. 299 Sauter, R. 81 Scott, R.M. 39,47,299 Shapiro, K.N. 8 Shrieve, D.C 197 Slater, J. 96 Squires, L.A. 303 Stachniak, J.B. 266 Stanley, P. 1 Steinbok, P. 141,255 Steinhardt, G. 96 Sutton, LN. 81,298

Tai, D. 289 Tarbell, N.J. 197 Thompson, D.N.P. 235 Tippets, R. 147 Trauner, D. 248 Tu, Y.-K. 158 Turrisi, A.T. 65

Walker,|M.L. 114,147,280 Wang, Z. 81 Waiters, G.V. 167 Weiner, H.L 309 Williams,_D. 96 Williams,|S. 241 Winston,|K.R. 228,241 Wisoff, J.H. 1,251,303,309 Wyllie, E. 181

Yachnis, A.T. 214 Yazbak, P.A. 15

Zeltzer, P. 1 Zide, B. Ill Zimmerman, R. 81 